\title{
A LÉVY PROCESS REFLECTED AT A POISSON AGE PROCESS
}

\author{
OFFER KELLA, ${ }^{*}$ The Hebrew University of Jerusalem \\ ONNO BOXMA, ${ }^{* *}$ EURANDOM and Eindhoven University of Technology \\ MICHEL MANDJES, ${ }^{* * *}$ CWI and The University of Amsterdam
}

\begin{abstract}
We consider a Lévy process with no negative jumps, reflected at a stochastic boundary that is a positive constant multiple of an age process associated with a Poisson process. We show that the stability condition for this process is identical to the one for the case of reflection at the origin. In particular, there exists a unique stationary distribution that is independent of initial conditions. We identify the Laplace-Stieltjes transform of the stationary distribution and observe that it satisfies a decomposition property. In fact, it is a sum of two independent random variables, one of which has the stationary distribution of the process reflected at the origin, and the other the stationary distribution of a certain clearing process. The latter is itself distributed as an infinite sum of independent random variables. Finally, we discuss the tail behavior of the stationary distribution and in particular observe that the second distribution in the decomposition always has a light tail.
\end{abstract}

Keywords: Lévy process; storage process; decomposition; queue with server vacations; secondary input

2000 Mathematics Subject Classification: Primary 60K05

Secondary $60 \mathrm{~K} 25$

\section{Introduction}

In various fields like queueing and risk theory it is natural to study a Lévy process (see, e.g. [3]) that is reflected at the origin. In this paper we study a Lévy process that has no negative jumps and is reflected at a stochastic lower barrier. This barrier is a straight line that drops to 0 after exponentially distributed time periods and then increases again linearly at rate $a$. It can also be viewed as a clearing process that increases linearly at some fixed rate, $a$, and at Poisson epochs drops to 0 (i.e. clears all the content from the system).

Applications in which such reflected Lévy processes are natural are models where, in addition to the input and output mechanism, there is a constant input that is not available on a liquid basis, but rather can be used only after some maturity date has been reached. In our case this maturity date is exponentially distributed. For example, consider the combined behavior of two financial accounts, namely a daily account and a savings account. The content of the daily account behaves like a Lévy process. The content of the savings account grows linearly at rate

\footnotetext{
Received 19 October 2005; revision received 4 December 2005.

* Postal address: Department of Statistics, The Hebrew University of Jerusalem, Mount Scopus, Jerusalem 91905, Israel. Email address: offer.kella@ huji.ac.il

** Postal address: Department of Mathematics and Computer Science, Eindhoven University of Technology, PO Box 513, 5600 MB Eindhoven, The Netherlands. Email address: boxma@win.tue.nl

*** Postal address: CWI, PO Box 94079, 1090 GB Amsterdam, The Netherlands.

Email address: michel.mandjes@cwi.nl
} 
$a$, and is moved to the daily account at exponentially distributed intervals. It is forbidden to let the daily account become negative. The combined level of the two accounts then behaves like a Lévy process reflected at a stochastic lower barrier.

We compute the stationary distribution of such a reflected Lévy process (when it exists) and observe that it satisfies a decomposition property. That is, it is the distribution of a sum of two independent random variables. The first has the stationary distribution of a Lévy process with no negative jumps and reflection at the origin, and the second is an independent, infinite sum of random variables that corresponds to the stationary distribution of a certain clearing process associated with the local time of the process.

The paper is organized in the following way. In Section 2 we consider the Lévy process, $W$, reflected at the above-described stochastic lower barrier, or age process, $A$. We determine necessary and sufficient conditions for the two-dimensional process $(W, A)$ to have a unique stationary distribution and a limiting distribution that is independent of initial conditions (see Theorem 1). In Section 3 we first consider the process $Z$, a Lévy process reflected at the origin, and its local time process, $L$. From the joint distribution of $Z(T), L(T)$, and $T$, where $T$ is exponentially distributed and independent of the Lévy process (see Theorem 2), the stationary distribution of $W$ is derived, in Section 3 (see Theorem 3). We show in particular that it satisfies a decomposition property, which is discussed in Section 4. The tail behavior of the stationary distribution of $W$ is analyzed in Section 5 (see Theorem 4), exploiting detailed knowledge of the two parts of the decomposition.

\section{Stability condition}

Let $X$ be a right-continuous Lévy process that is not almost surely nondecreasing (i.e. not a subordinator), starts at an arbitrary initial value, has no negative jumps, and has exponent $\phi(\alpha)=\log \mathrm{E}\left[\mathrm{e}^{-\alpha X(1)}\right]$. Now suppose that the Lévy process is reflected along a boundary of the form $A(t)=A(0) \mathbf{1}_{\left\{T_{1}>t\right\}}+a\left(t-T_{N(t)}\right)$, where $a>0 ; N$ is a Poisson process with rate $\lambda$ and arrival epochs $T_{n}$, and is independent of the Lévy process; and $A(0)$ is some nonnegative random variable (possibly 0 ) that is independent of everything else. That is, at each point the boundary increases linearly at a rate $a$, and at the arrival epochs of the Poisson process it jumps back to 0 . However, at time 0 it may start at some arbitrary nonnegative value.

The appropriate stability condition for such a Lévy process is that $\phi^{\prime}(0)>0$. To see this we observe that the process is of the form $W(t)=W(0)+X(t)+L(t)$, where $L(0)=0, L$ is nondecreasing, $W(t) \geq A(t)$, and $L$ can increase only at points, $t$, for which $W(t)=A(t)$. Thus, $W(t)-A(t)=W(0)+X(t)-A(t)+L(t)$ is a reflected process with driver $X(t)-A(t)$. Therefore,

$$
W(t)-A(t)=W(0)+X(t)-A(t)-\inf _{0 \leq s \leq t}(W(0)+X(s)-A(s)) \wedge 0 .
$$

Since $X(t) / t \rightarrow-\phi^{\prime}(0)$ and $A(t) / t \rightarrow 0$, both almost surely, it follows that there exists some finite $T>T_{1}$ for which $W(0)+X(T)-A(T) \leq 0$. Hence, for $t \geq T$ the minimization with 0 can be omitted. After canceling $W(0)$ on the right-hand side and $A(t)$ from both sides, for $t>T$ we have

$$
W(t)=X(t)-\inf _{0 \leq s \leq t}(X(s)-A(s)) .
$$

Also, since $X(t)-A(t) \rightarrow-\infty$ almost surely, for sufficiently large $t$ the infimum depends on values of $s$ that are larger than $T_{1}$; thus, for such values of $t,(W(t), A(t))$ does not depend on the value of $A(0)$. This means that, for any initial $W(0)$ and $A(0)$, the process $(W(t), A(t))$ 
can be coupled with the process that starts with $W(0)=A(0)=0$, and, thus, if a limiting distribution exists, it does not depend on initial conditions.

To show that a limiting distribution exists we start the process with $W(0)=A(0)$, where $A(0) \stackrel{\mathrm{D}}{=} \operatorname{Exp}(\lambda / a)$ (and ' $\stackrel{\mathrm{D}}{=}$ ' denotes equality in distribution). With this choice, $A(t)$ becomes a stationary process. In fact, $A(t) / a$ is a stationary version of the age process associated with the Poisson process. Let us first extend both the Lévy process, $X$, and $A$ to the whole real line. Since $W(0)-A(0)=0$ we have

$$
W(t)=X(t)-\inf _{0 \leq s \leq t}(X(s)-A(s))=\sup _{0 \leq s \leq t}(X(t)-X(t-s)+A(t-s))
$$

and, upon shifting by $t$ and recalling the stationarity of $A$ and the (strong) stationary increments property of $X$, we obtain

$$
W(t) \stackrel{\mathrm{D}}{=} S(t):=\sup _{0 \leq s \leq t}(-X(-s)+A(-s)),
$$

and in fact $(W(t), A(t)) \stackrel{\mathrm{D}}{=}(S(t), A(0))$. In particular, $W(t)$ is stochastically increasing.

Clearly, $\{-X(-s), s \geq 0\}$ is a left-continuous version of a Lévy process with the same exponent as $X$, and $A(-s)$ is a left-continuous version of the process $R(t)=a\left(T_{N(t)+1}-t\right)$. Note that $R / a$ is the residual lifetime process associated with $N$. As the supremum does not depend on whether we take the right- or left-continuous version (in particular, the endpoints are almost surely points of continuity), we see that $(W(t), A(t))$ has the same distribution as

$$
\left(\sup _{0 \leq s \leq t}(X(s)+R(s)), R(0)\right),
$$

where $X$ and $R$ are independent processes. Recalling that $X(t) / t \rightarrow-\phi^{\prime}(0)<0$ and noting that $R(t) / t \rightarrow 0$, both almost surely, it follows that $X(t)+R(t) \rightarrow-\infty$ almost surely and, thus, that $W(t)$ converges in distribution to $\sup _{s \geq 0}(X(s)+R(s))$, which is an almost-surely finite random variable.

In fact, we observe that the process $\left(W^{*}, A\right)$, in which

$$
\begin{aligned}
W^{*}(t) & =\sup _{s \leq 0}(X(t)-X(t+s)+A(t+s)) \\
& =X(t)-\inf _{s \leq t}(X(s)-A(s)) \\
& =W^{*}(0)+X(t)-\inf _{0 \leq s \leq t}\left(W^{*}(0)+X(s)\right)^{-},
\end{aligned}
$$

is a stationary version of $W$. Also, we observe that if $\phi^{\prime}(0) \leq 0$ then $W$ is above the process $W(0)+X(t)-\inf _{0 \leq s \leq t}(W(0)+X(s))^{-}$, which is not positive recurrent; thus, neither is $W$. We summarize these results in the following theorem.

Theorem 1. The Markov process $\{(W(t), A(t)), t \geq 0\}$ is positive Harris recurrent (and thus has a unique stationary distribution and a limiting distribution independent of initial conditions) if and only if $\phi^{\prime}(0)>0$.

Now let $Z(t)=W(t)-a t=W(0)+X(t)-a t+L(t)$. Until time $T_{1},(Z, L)$ is a reflected Lévy process with driving process $X(t)-a t$, having an exponent $\tilde{\phi}(\alpha)=\phi(\alpha)+a \alpha$, which satisfies $\tilde{\phi}^{\prime}(0)>a$. Thus, $W(T)$ is distributed like $W(0)$ if and only if $Z(T)+a T$ is distributed like $Z(0)$. Owing to the PASTA (Poisson Arrivals See Time Averages) property, $W(0) \stackrel{\mathrm{D}}{=} W(T)$ if and only if $W(0)$ has the time-stationary distribution of $W$. In the following section we will find the distribution of $Z(0)$ for which $Z(T)+a T \stackrel{\mathrm{D}}{=} Z(0)$. 


\section{When does $Z(0) \stackrel{\mathrm{D}}{=} Z(T)+a T$ hold?}

As described in the previous section, the key to computing the stationary distribution of $W$ is finding an initial distribution such that $Z(0)$ and $Z(T)+a T$ are identically distributed. For ease of notation we will use the notation $\phi$ rather than $\tilde{\phi}$ (where $\tilde{\phi}(\alpha)=\phi(\alpha)+a \alpha$ throughout); hence, we assume that $\phi^{\prime}(0)>a$. Also, here we let E denote the expectation associated with the initial distribution that we are seeking. Our starting point is Theorem 3.10 of [2, p. 259], which we rewrite as Theorem 2, below. In [2] the assumption that $X$ is not a subordinator is missing from the statement of Theorem 3.10, but it is evident from the proof that it is assumed.

Theorem 2. Let $X$ be a Lévy process that has no negative jumps, is not a subordinator, and has exponent $\phi$, and let $\psi\left(\alpha_{0}\right)=\inf \left\{\alpha: \phi(\alpha)>\alpha_{0}\right\}$ for $\alpha_{0} \geq 0$. Then, for $T \stackrel{\mathrm{D}}{=} \operatorname{Exp}(\lambda)$, which is independent of $X$,

$$
\begin{aligned}
& \mathrm{E}_{z}\left[\mathrm{e}^{-(\alpha Z(T)+\beta L(T)+\gamma T)}\right] \\
& \quad=\left(\mathrm{e}^{-\psi(\lambda+\gamma) z} \frac{1-\alpha / \psi(\lambda+\gamma)}{1-\phi(\alpha) /(\lambda+\gamma)} \frac{\psi(\lambda+\gamma)}{\psi(\lambda+\gamma)+\beta}+\frac{\mathrm{e}^{-\alpha z}-\mathrm{e}^{-\psi(\lambda+\gamma) z}}{1-\phi(\alpha) /(\lambda+\gamma)}\right) \frac{\lambda}{\lambda+\gamma}
\end{aligned}
$$

for all nonnegative $z, \alpha, \beta$, and $\gamma$.

Set $\gamma=\alpha a$ and $\beta=0$ in (1) and simplify, to obtain

$$
(\phi(\alpha)-\alpha a) \frac{\mathrm{E}\left[\mathrm{e}^{-\alpha Z(0)}\right]}{\alpha}=\lambda \frac{\mathrm{E}\left[\mathrm{e}^{-\psi(\lambda+\alpha a) Z(0)}\right]}{\psi(\lambda+\alpha a)} ;
$$

in particular, by taking $\alpha \downarrow 0$, we have

$$
\phi^{\prime}(0)-a=\lambda \frac{\mathrm{E}\left[\mathrm{e}^{-\psi(\lambda) Z(0)}\right]}{\psi(\lambda)} .
$$

Since the right-hand side is positive, we see that a necessary condition for the existence of a distribution of $Z(0)$ for which $Z(T)+a T \stackrel{\mathrm{D}}{=} Z(0)$ is indeed that $\phi^{\prime}(0)>a$. By letting $f(\lambda, a, \alpha)=\mathrm{E}\left[\mathrm{e}^{-\alpha Z(0)}\right]$, where $Z(0)$ has the stationary distribution that we seek, and noting that $\psi^{\prime}(0)=1 / \phi^{\prime}(0)$, we obtain

$$
\lim _{\lambda \downarrow 0} f(\lambda, a, \psi(\lambda))=1-\frac{a}{\phi^{\prime}(0)}<1
$$

It may seem as if (3) implies that $\mathrm{P}[Z(0)<\infty]<1$, but it does not, as the distribution of $Z(0)$ depends on $\lambda$.

If we let $a=0$ then we obtain

$$
\phi^{\prime}(0)=\frac{\lambda}{\psi(\lambda)} \mathrm{E}\left[\mathrm{e}^{-\psi(\lambda) Z(0)}\right]
$$

and, thus, $\mathrm{E}\left[\mathrm{e}^{-\alpha Z(0)}\right]=\phi^{\prime}(0) \alpha / \phi(\alpha)$ (which is the generalized Pollaczek-Khinchin formula) as expected, since then $Z$ is reflected at the origin and, in this case, the continuous-time process has the same stationary distribution as the process sampled at Poisson epochs.

Now let $h(\alpha)=\psi(\lambda+a \alpha)$ and assume that $\phi^{\prime}(0)>a$. Then, since $\psi$ is concave we have $h^{\prime}(0)=a \psi^{\prime}(\lambda)<a \psi^{\prime}(0)=a / \phi^{\prime}(0)<1$, and since $h$ is concave it is Lipshitz continuous, with $|h(\alpha)-h(\beta)| \leq h^{\prime}(0)|\alpha-\beta|$, and is thus a contraction. Writing $h^{0}(\alpha)=\alpha$ and 
$h^{i+1}(\alpha)=h\left(h^{i}(\alpha)\right)$, we thus find that, for any $\alpha \geq 0, h^{i}(\alpha) \rightarrow \alpha^{*}$ as $i \rightarrow \infty$, with $\alpha^{*}=h\left(\alpha^{*}\right)$. That is, if we let $\phi_{a}(\alpha):=\phi(\alpha)-a \alpha$ and let $\psi_{a}(\beta)$ be its inverse, then it is easy to check that $\alpha^{*}=\psi_{a}(\lambda)$.

With this in mind, (2) may be rewritten as

$$
\mathrm{E}\left[\mathrm{e}^{-\alpha Z(0)}\right]=\frac{\lambda}{\phi_{a}(\alpha)} \frac{\alpha \mathrm{E}\left[\mathrm{e}^{-h(\alpha) Z(0)}\right]}{h(\alpha)},
$$

which, for $n \geq 1$, immediately leads to

$$
\mathrm{E}\left[\mathrm{e}^{-\alpha Z(0)}\right]=\frac{\lambda \alpha \mathrm{E}\left[\mathrm{e}^{-h^{n+1}(\alpha) Z(0)}\right]}{\phi_{a}(\alpha) h^{n+1}(\alpha)} \prod_{i=1}^{n} \frac{\lambda}{\phi_{a}\left(h^{i}(\alpha)\right)} .
$$

Upon substituting $\alpha=0$, we have

$$
1=\frac{\lambda \mathrm{E}\left[\mathrm{e}^{-h^{n+1}(0) Z(0)}\right]}{\phi_{a}^{\prime}(0) h^{n+1}(0)} \prod_{i=1}^{n} \frac{\lambda}{\phi_{a}\left(h^{i}(0)\right)} .
$$

Therefore,

$$
\mathrm{E}\left[\mathrm{e}^{-\alpha Z(0)}\right]=\frac{\alpha \phi_{a}^{\prime}(0)}{\phi_{a}(\alpha)} \frac{h^{n+1}(0) \mathrm{E}\left[\mathrm{e}^{-h^{n+1}(\alpha) Z(0)}\right]}{h^{n+1}(\alpha) \mathrm{E}\left[\mathrm{e}^{-h^{n+1}(0) Z(0)}\right]} \prod_{i=1}^{n} \frac{\phi_{a}\left(h^{i}(0)\right)}{\phi_{a}\left(h^{i}(\alpha)\right)} .
$$

Observe that $h^{n+1}(\alpha)$ and $h^{n+1}(0)$ both converge to $\alpha^{*}=\psi_{a}(\lambda)$ as $n \rightarrow \infty$. Also, since $\phi_{a}^{\prime}(0)=\phi^{\prime}(0)-a>0$, it follows that $\phi_{a}$ is an increasing function on $[0, \infty)$. By induction, the $h^{i}$ are also increasing and, thus, for every $i, \phi_{a}\left(h^{i}(0)\right) / \phi_{a}\left(h^{i}(\alpha)\right)<1$ for all $\alpha>0$. Thus, the product converges to a proper limit and we obtain the following decomposition result.

Theorem 3. For all $\alpha \geq 0$,

$$
\mathrm{E}\left[\mathrm{e}^{-\alpha Z(0)}\right]=\frac{\phi_{a}^{\prime}(0) \alpha}{\phi_{a}(\alpha)} \prod_{i=1}^{\infty} \frac{\phi_{a}\left(h^{i}(0)\right)}{\phi_{a}\left(h^{i}(\alpha)\right)} .
$$

\section{Interpretation of the decomposition result}

This section is devoted to understanding the right-hand side of the decomposition (4), and, in particular, to showing that it is the Laplace-Stieltjes transform (LST) of a proper random variable.

It is easy to check that $\phi_{a}\left(h^{i}(\alpha)\right)=\lambda+\left(h^{i-1}(\alpha)-h^{i}(\alpha)\right) a$, and we may thus rewrite (4) as

$$
\mathrm{E}\left[\mathrm{e}^{-\alpha Z(0)}\right]=\frac{\phi_{a}^{\prime}(0) \alpha}{\phi_{a}(\alpha)} \prod_{i=1}^{\infty} \frac{\lambda+\left(h^{i-1}(0)-h^{i}(0)\right) a}{\lambda+\left(h^{i-1}(\alpha)-h^{i}(\alpha)\right) a} .
$$

In particular, recalling that $\phi_{a}\left(\alpha^{*}\right)=\lambda$ and $h^{i}\left(\alpha^{*}\right)=\alpha^{*}$, we have

$$
\mathrm{E}\left[\mathrm{e}^{-\alpha^{*} Z(0)}\right]=\frac{\phi_{a}^{\prime}(0) \alpha^{*}}{\lambda} \prod_{i=1}^{\infty}\left(1+\left(h^{i-1}(0)-h^{i}(0)\right) \frac{a}{\lambda}\right) .
$$


Since $\alpha<\alpha^{*}=\psi_{a}(\lambda)$ is equivalent to $\phi(\alpha)-a \alpha<\lambda$, which is in turn equivalent to $\alpha<\psi(\lambda+a \alpha)=h(\alpha)$, we conclude that $h^{i}(\alpha)$ is an increasing sequence. Similarly, when $\alpha>\alpha^{*}, h^{i}(\alpha)$ is a decreasing sequence.

To show that the right-hand side of (5) is the LST of a proper distribution, it suffices to show that the second term (the product term) converges to 1 as $\alpha \downarrow 0$. To see this we first give the following simple lemma.

Lemma 1. Let $a_{i}:[0, \infty) \rightarrow[0, \infty)$ be such that $a_{i}(\alpha) \rightarrow 0$ as $\alpha \downarrow 0, a_{i}(\alpha) \leq a_{i} b(\alpha)$, where $b(\alpha)$ is bounded in some neighborhood of 0 , and $\sum_{i=1}^{\infty} a_{i}<\infty$. Then

$$
\lim _{\alpha \downarrow 0} \prod_{i=1}^{\infty} \frac{1}{1+a_{i}(\alpha)}=1
$$

Proof. For a given $\varepsilon>0$, choose an $N$ such that $\sum_{i=N+1}^{\infty} a_{i}<\varepsilon$. Since

$$
1+a_{i}(\alpha) \leq 1+a_{i} b(\alpha) \leq \mathrm{e}^{a_{i} b(\alpha)}
$$

we have

$$
\prod_{i=N+1}^{\infty} \frac{1}{1+a_{i}(\alpha)} \geq \exp \left(-b(\alpha) \sum_{i=N+1}^{\infty} a_{i}\right) \geq \mathrm{e}^{-b(\alpha) \varepsilon} \geq 1-b(\alpha) \varepsilon
$$

Clearly,

$$
\lim _{\alpha \downarrow 0} \prod_{i=1}^{N} \frac{1}{1+a_{i}(\alpha)}=1
$$

for each $N$, and, thus, as $\varepsilon$ is arbitrary and $b$ is bounded in some neighborhood of 0 , the proof is complete.

Now observe that if we let

$$
\frac{\lambda+\left(h^{i-1}(0)-h^{i}(0)\right) a}{\lambda+\left(h^{i-1}(\alpha)-h^{i}(\alpha)\right) a}=\frac{1}{1+a_{i}(\alpha)}
$$

then

$$
a_{i}(\alpha)=\frac{(a / \lambda)\left(h^{i-1}(\alpha)-h^{i-1}(0)+h^{i}(0)-h^{i}(\alpha)\right)}{1+(a / \lambda)\left(h^{i-1}(0)-h^{i}(0)\right)} .
$$

Recall that $h$ is contracting, implying that $\left|h^{n}(\alpha)-h^{n}(0)\right| \leq \rho^{n} \alpha$, where $\rho=h^{\prime}(0)=$ $a \psi^{\prime}(\lambda)<1$. In particular, $\left|h^{i-1}(0)-h^{i}(0)\right| \leq \rho^{i-1} h(0)$. Therefore,

$$
a_{i}(\alpha) \leq \frac{(a / \lambda)(1+\rho) \rho^{i-1} \alpha}{1-(a / \lambda) \rho^{i-1} h(0)} .
$$

Let $i_{0}$ be such that $(a / \lambda) \rho^{i_{0}-1} h(0)<\frac{1}{2}, a_{i}=\rho^{i-1}$, and

$$
b(\alpha)=2 \frac{a}{\lambda}(1+\rho) \alpha \max _{1 \leq i<i_{0}} \frac{a_{i}(\alpha)}{\rho^{i-1}} .
$$

The conditions of Lemma 1 are then satisfied and, thus, if the right-hand side of (5) is an LST, it is the LST of a proper distribution. 
Let us now argue that it is indeed an LST.

Lemma 2. We have $\mathrm{e}^{-h^{n}(\alpha) z}=\mathrm{E}\left[\mathrm{e}^{-\left(\lambda \xi_{n}(z)+\alpha \eta_{n}(z)\right)}\right]$, where $\left(\xi_{n}, \eta_{n}\right)$ is a two-dimensional nondecreasing Lévy process with exponent, $f_{n}$, satisfying $f_{n}(x, y)=\psi\left(x+a f_{n-1}(x, y)\right)$, so that $f_{n}(\lambda, \alpha)=h^{n}(\alpha)$. Thus, for every nonnegative random variable $\zeta$,

$$
\frac{\mathrm{E}\left[\mathrm{e}^{-h^{n}(\alpha) \zeta}\right]}{\mathrm{E}\left[\mathrm{e}^{-h^{n}(0) \zeta}\right]}=\mathrm{E}\left[\mathrm{e}^{-\alpha \eta_{n}(\zeta)} \mid \xi_{n}(\zeta)<T\right],
$$

where $T$ is an independent exponential random variable with rate $\lambda$. Therefore, the left-hand side is an LST for any distribution of $\zeta$.

Proof. Assume that $X_{i}$ are independent Lévy processes all distributed like $X$. For $z \geq 0$, let $\tau_{i}(z)=\inf \left\{t: X_{i}(t)+z=0\right\}$. Then it is well known that the $\tau_{i}$ are (independent) subordinators (nondecreasing Lévy processes) with $\mathrm{E}\left[\mathrm{e}^{-\alpha \tau_{i}(z)}\right]=\mathrm{e}^{-\psi(\alpha) z}$. In particular,

$$
\begin{aligned}
\mathrm{e}^{-h^{1}(\alpha) z}=\mathrm{e}^{-\psi(\lambda+a \alpha) z} & =\mathrm{E}\left[\mathrm{e}^{-(\lambda+a \alpha) \tau_{1}(z)}\right], \\
\mathrm{e}^{-h^{2}(\alpha) z}=\mathrm{e}^{-\psi\left(\lambda+a h^{1}(\alpha)\right) z} & =\mathrm{E}\left[\mathrm{e}^{-\left(\lambda+a h^{1}(\alpha)\right) \tau_{2}(z)}\right] \\
& =\mathrm{E}\left[\exp \left(-\left[\lambda \tau_{2}(z)+(\lambda+a \alpha) \tau_{1}\left(a \tau_{2}(z)\right)\right]\right)\right] ;
\end{aligned}
$$

by induction it is easy to check that

$$
\mathrm{e}^{-h^{n}(\alpha) z}=\mathrm{E}\left[\exp \left(-\left(\frac{\lambda}{a} \xi_{n}(z)+\alpha \eta_{n}(z)\right)\right)\right]
$$

where $\eta_{n}(z)=\eta_{n-1}\left(a \tau_{n}(z)\right)$ with $\eta_{0}(z)=z$, and $\xi_{n}(z)=\tau_{n}(z)+\xi_{n-1}\left(a \tau_{n}(z)\right)$ with $\xi_{0}(z)=0$. It is easy to check that $\left(\eta_{1}, \ldots, \eta_{n}\right)$ is a multidimensional Lévy process and that $\left(\xi_{n}, \eta_{n}\right)$ is thus a two-dimensional one. Obtaining the form of the exponent is straightforward upon observing that $f_{n}(\lambda, \alpha)=h^{n}(\alpha)$.

Lemma 3. Let $g(\alpha):=\alpha-a \psi(\alpha)$ and $\tau(z):=\inf \{t: X(t)+z=0\}$, and set $J(t):=a \tau(t)-t$. Then $J$ is a Lévy process with no negative jumps and Laplace exponent $g$. If $\phi^{\prime}(0)>a$ then $g^{\prime}(0)>0$, and, thus, $\mathrm{E}\left[\mathrm{e}^{-\alpha \zeta}\right]=g^{\prime}(0) \alpha / g(\alpha)$ is the LST of the stationary distribution associated with $J$, reflected at the origin.

Proof. It is well known that $\tau(\cdot)$ is a subordinator with exponent $-\psi$. Thus, the exponent of $J$ is clearly $\alpha-a \psi(\alpha)=g(\alpha)$. Now note that $g^{\prime}(0)=1-a \psi^{\prime}(0)=1-a / \phi^{\prime}(0)>0$, which is the condition under which the generalized Pollaczek-Khinchin formula for the stationary distribution is valid.

It is now easy to check that $\phi_{a}\left(h^{n}(\alpha)\right)=g\left(\lambda+a h^{n-1}(\alpha)\right)$ for $n \geq 1$, where we recall that $h^{0}(\alpha)=\alpha$. Thus, we can write

$$
\frac{\phi_{a}\left(h^{n}(0)\right)}{\phi_{a}\left(h^{n}(\alpha)\right)}=\frac{g\left(\lambda+a h^{n-1}(0)\right)\left(\lambda+a h^{n-1}(\alpha)\right)}{g\left(\lambda+a h^{n-1}(\alpha)\right)\left(\lambda+a h^{n-1}(0)\right)} \cdot \frac{\lambda+a h^{n-1}(0)}{\lambda+a h^{n-1}(\alpha)} .
$$

Applying Lemma 2 with $T=T_{1}$ and $\zeta=a T_{2}$, where $T_{1}, T_{2} \stackrel{\mathrm{D}}{=} \operatorname{Exp}(\lambda)$ are independent, we have

$$
\frac{\lambda+a h^{n-1}(0)}{\lambda+a h^{n-1}(\alpha)}=\frac{\mathrm{E}\left[\mathrm{e}^{-h^{n-1}(\alpha) a T_{2}}\right]}{\mathrm{E}\left[\mathrm{e}^{-h^{n-1}(0) a T_{2}}\right]}=\mathrm{E}\left[\mathrm{e}^{-\alpha \eta_{n-1}\left(a T_{2}\right)} \mid \xi_{n-1}\left(a T_{2}\right)<T_{1}\right] .
$$


It is easy similarly to check that if $\zeta$ has the stationary distribution of the reflected version of $J$ from Lemma 3, then the first term on the right-hand side of (6) can be written as

$$
\begin{aligned}
\frac{\mathrm{E}\left[\mathrm{e}^{-\left(\lambda+a h^{n-1}(\alpha)\right) \zeta}\right]}{\mathrm{E}\left[\mathrm{e}^{-\left(\lambda+a h^{n-1}(0)\right) \zeta}\right]} & =\frac{\mathrm{E}\left[\exp \left(-\left[\lambda\left(\zeta+\xi_{n-1}(a \zeta)\right)+\alpha \eta_{n-1}(a \zeta)\right]\right)\right]}{\mathrm{E}\left[\mathrm{e}^{-\lambda\left(\zeta+\xi_{n-1}(a \zeta)\right)}\right]} \\
& =\mathrm{E}\left[\mathrm{e}^{-\alpha \eta_{n-1}(a \zeta)} \mid \zeta+\xi_{n-1}(a \zeta)<T\right] .
\end{aligned}
$$

We therefore have the following corollary.

Corollary 1. We have

$$
\frac{\phi_{a}\left(h^{n}(0)\right)}{\phi_{a}\left(h^{n}(\alpha)\right)}=\mathrm{E}\left[\mathrm{e}^{-\alpha \eta_{n-1}(a \zeta)} \mid \zeta+\xi_{n-1}(a \zeta)<T\right] \mathrm{E}\left[\mathrm{e}^{-\alpha \eta_{n-1}\left(a T_{2}\right)} \mid \xi_{n-1}\left(a T_{2}\right)<T_{1}\right] .
$$

The left-hand side is thus the LST of a proper random variable.

Since $\phi_{a}^{\prime}(0) \alpha / \phi_{a}(\alpha)$ is the LST of the stationary distribution of

$$
Z_{a}(t)=X(t)-a t-\inf _{0 \leq s \leq t}(X(s)-a s)
$$

(recalling that $\left.\phi^{\prime}(0)>a\right)$, it is now clear that the right-hand side of (4) is indeed the LST of a proper distribution on $[0, \infty)$.

There are, however, alternative ways to interpret the decomposition of Theorem 3. One that adds insight is the following. Apply the martingale of [7], and observe that $W(t)=a t$ at points of increase of $L(t)$, for $0 \leq t \leq T$. As a consequence, with E denoting the expectation associated with the stationary version, we have

$$
\phi(\alpha) \mathrm{E}\left[\int_{0}^{T} \mathrm{e}^{-\alpha W(s)} \mathrm{d} s\right]=\mathrm{E}\left[\mathrm{e}^{-\alpha W(T)}\right]-\mathrm{E}\left[\mathrm{e}^{-\alpha W(0)}\right]+\alpha \mathrm{E}\left[\int_{0}^{T} \mathrm{e}^{-\alpha a s} \mathrm{~d} L(s)\right] .
$$

Observe that

$$
\begin{aligned}
\lambda \mathrm{E}\left[\int_{0}^{T} \mathrm{e}^{-\alpha W(s)} \mathrm{d} s\right] & =\lambda \mathrm{E}\left[\int_{0}^{\infty} \mathrm{e}^{-\alpha W(s)} \mathbf{1}_{\{T>s\}} \mathrm{d} s\right] \\
& =\int_{0}^{\infty} \mathrm{E}\left[\mathrm{e}^{-\alpha W(s)}\right] \lambda \mathrm{e}^{-\lambda s} \mathrm{~d} s \\
& =\mathrm{E}\left[\mathrm{e}^{-\alpha W(T)}\right] .
\end{aligned}
$$

Furthermore, since $W(0) \stackrel{\text { D }}{=} W(T)$, we have

$$
\mathrm{E}\left[\mathrm{e}^{-\alpha W(0)}\right]=\frac{\lambda \alpha}{\phi(\alpha)} \mathrm{E}\left[\int_{0}^{T} \mathrm{e}^{-\alpha a t} \mathrm{~d} L(t)\right]
$$

and, upon setting $\alpha=0$, we obtain $\mathrm{E}[L(T)]=\phi^{\prime}(0) / \lambda$ and, in particular,

$$
\mathrm{E}\left[\mathrm{e}^{-\alpha W(0)}\right]=\frac{\phi^{\prime}(0) \alpha}{\phi(\alpha)} \frac{\mathrm{E}\left[\int_{0}^{T} \mathrm{e}^{-\alpha a t} \mathrm{~d} L(t)\right]}{\mathrm{E}[L(T)]} .
$$

The first term in this decomposition is the generalized Pollaczek-Khinchin formula associated with the stationary distribution of $X$ reflected at the origin. It is easy to check that the second term in the decomposition on the right is the LST of the distribution

$$
F(x)=\frac{\mathrm{E}[L(T \wedge x / a)]}{\mathrm{E}[L(T)]}
$$


where the expectation is taken when $W$ is initiated with its stationary distribution. That is, consider a clearing process with cycles distributed like $T$ and which, during a clearing cycle, behaves like $L(t)$, where $W(0)$ has the stationary distribution; then

$$
F(a x)=\frac{\mathrm{E}[L(T \wedge x)]}{\mathrm{E}[L(T)]}
$$

is well known to be the stationary distribution of this process.

We mention that in [6] related decompositions were studied. In the framework of that paper (a reflected Lévy process with additional jumps), the stationary distribution is also a convolution of two or more distributions, one of which is the stationary distribution of the process reflected at the origin.

\section{Tail behavior}

In this section we analyze the tail behavior of the distribution whose LST is the infinite product in (4). More specifically, we show that this tail is exponential with decay rate $\lambda / a$, and we compute the prefactor. To obtain the full tail behavior of $Z(0)$, this needs to be combined with the tail behavior associated with the LST $\phi_{a}^{\prime}(0) \alpha / \phi_{a}(\alpha)$; see Theorem 3 . These asymptotics have been studied in detail before. For the Cramér case, in which the tail is exponential, see in particular [4]. The subexponential case, in which the Lévy process is an independent sum of a Brownian motion and a subordinator, has been studied in, e.g. [1]. Recently, Dieker [5] produced a more complete study of the tail behavior of the supremum of a Lévy process. This reference also contains an overview of the related literature.

Consider the infinite product in (4). Since $h^{n}(\alpha)=h^{n-1}(h(\alpha))=h^{n-1}(\psi(\lambda+a \alpha))$, we have $h^{n}(\alpha) \geq h^{n-1}(0)$ for $\alpha \geq-\lambda / a$. Thus,

$$
\prod_{n=2}^{\infty} \frac{\phi_{a}\left(h^{n}(0)\right)}{\phi_{a}\left(h^{n}(\alpha)\right)} \leq \prod_{n=2}^{\infty} \frac{\phi_{a}\left(h^{n}(0)\right)}{\phi_{a}\left(h^{n-1}(0)\right)}=\frac{\phi_{a}\left(\alpha^{*}\right)}{\phi_{a}(\psi(\lambda))}=\frac{\lambda}{\lambda-a \psi(\lambda)},
$$

with equality when $\alpha=-\lambda / a$. For the first term in the product in (4) we have

$$
\frac{\phi_{a}(h(0))}{\phi_{a}(h(\alpha))}=\frac{g(\lambda)}{g(\lambda+a \alpha)}=\frac{\lambda}{\lambda+a \alpha} \frac{g(\lambda)(\lambda+a \alpha)}{\lambda g(\lambda+a \alpha)},
$$

where, due to Lemma 3, the second factor of the right-most expression is the LST of a proper random variable. Let $V_{1} \stackrel{\mathrm{D}}{=} \operatorname{Exp}(\lambda / a)$ and let $V_{2}$ be independent of $V_{1}$ with

$$
\mathrm{E}\left[\mathrm{e}^{-\alpha V_{2}}\right]=\frac{g(\lambda)(\lambda+a \alpha)}{\lambda g(\lambda+a \alpha)} \prod_{n=2}^{\infty} \frac{\phi_{a}\left(h^{n}(0)\right)}{\phi_{a}\left(h^{n}(\alpha)\right)} .
$$

It is then easy to check, recalling the right-hand side of (7), that

$$
\mathrm{E}\left[\mathrm{e}^{(\lambda / a) V_{2}}\right]=\frac{g(\lambda)}{\lambda g^{\prime}(0)} \frac{\lambda}{\lambda-a \psi(\lambda)}=\frac{1}{1-a / \phi^{\prime}(0)} .
$$

Therefore, the infinite product in (4) is the LST of $V=V_{1}+V_{2}$, where $\mathrm{E}\left[\mathrm{e}^{\beta V_{2}}\right]<\infty$ and $V_{1} \stackrel{\mathrm{D}}{=} \operatorname{Exp}(\beta)$, with $\beta=\lambda / a$. Since

$$
\mathrm{P}\left[V_{2}>t\right] \leq \mathrm{e}^{-\beta t} \mathrm{E}\left[\mathrm{e}^{\beta V_{2}} \mathbf{1}_{\left\{V_{2}>t\right\}}\right],
$$


it follows by dominated convergence that $\mathrm{e}^{\beta t} \mathrm{P}\left[V_{2}>t\right] \rightarrow 0$ as $t \rightarrow \infty$. Now,

$$
\mathrm{e}^{\beta t} \mathrm{P}[V>t]=\mathrm{e}^{\beta t} \mathrm{E}\left[\mathrm{e}^{-\beta\left(t-V_{2}\right)^{+}}\right]=\mathrm{E}\left[\mathrm{e}^{\beta V_{2}} \mathbf{1}_{\left\{V_{2} \leq t\right\}}\right]+\mathrm{e}^{\beta t} \mathrm{P}\left[V_{2}>t\right]
$$

and taking $t \rightarrow \infty$ gives

$$
\mathrm{e}^{\beta t} \mathrm{P}[V>t] \rightarrow \mathrm{E}\left[\mathrm{e}^{\beta V_{2}}\right]
$$

Thus, in view of (8), as $t \rightarrow \infty$ we have

$$
\mathrm{P}[V>t] \sim \frac{\mathrm{e}^{-(\lambda / a) t}}{1-a / \phi^{\prime}(0)},
$$

where we write $f \sim g$ to mean that $f(t) / g(t) \rightarrow 1$ as $t \rightarrow \infty$. Because $V_{1} \stackrel{\mathrm{D}}{=} \operatorname{Exp}(\beta)$ and

$$
\frac{\mathrm{P}\left[V_{2}>t\right]}{\mathrm{P}\left[V_{1}>t\right]}=\mathrm{e}^{\beta t} \mathrm{P}\left[V_{2}>t\right] \rightarrow 0,
$$

(9) is in fact a special case of Lemma 2.1 of [8].

The above can be summarized as follows.

Theorem 4. Let $V$ be a random variable with LST $\prod_{i=1}^{\infty} \phi_{a}\left(h^{i}(0)\right) / \phi_{a}\left(h^{i}(\alpha)\right)$. Then, as $t \rightarrow \infty$,

$$
\mathrm{P}[V>t] \sim \frac{\mathrm{e}^{-(\lambda / a) t}}{1-a / \phi^{\prime}(0)} .
$$

Denote by $\bar{V}$ a random variable that has $\operatorname{LST} \phi_{a}^{\prime}(0) \alpha / \phi_{a}(\alpha)$, is independent of $V$, and is such that $Z(0)$ is distributed as $\bar{V}+V$; see Theorem 3. Theorem 4 implies that if $\bar{V}$ is subexponential, then $Z(0)$ is also subexponential and has the same tail behavior as $\bar{V}$. If the tail of $\bar{V}$ is exponential (as in the Cramér case mentioned above), with a decay rate that differs from $\lambda / a$, then the heavier tail dominates, and determines the decay rate of $Z(0)$; the prefactor can be computed in a similar way as above.

\section{Acknowledgements}

The authors are indebted to the referee for several useful remarks. This work was done while the first author visited EURANDOM and CWI as a Stieltjes visiting professor. The first author is also supported by grant $819 / 03$ from the Israel Science Foundation. The research of the other two authors was done within the framework of the BRICKS project and the European Network of Excellence Euro-NGI.

\section{References}

[1] Asmussen, S. (1998). Subexponential asymptotics for stochastic processes: extremal behavior, stationary distributions and first passage probabilities. Ann. Appl. Prob. 8, 354-374.

[2] Asmussen, S. (2003). Applied Probability and Queues (Appl. Math. (New York) 51), 2nd edn. Springer, New York.

[3] Bertoin, J. (1996). Lévy Processes. Cambridge University Press.

[4] Bertoin, J. and Doney, R. A. (1994). Cramér's estimate for Lévy processes. Statist. Prob. Lett. 21, $363-365$.

[5] Dieker, A. B. (2005). Applications of factorization embeddings for Lévy processes. Submitted. Available at http://www.cwi.nl/ ton.

[6] Kella, O. ANd Whitt, W. (1991). Queues with server vacations and Lévy processes with secondary jump input. Ann. Appl. Prob. 1, 104-117.

[7] Kella, O. and Whitt, W. (1992). Useful martingales for stochastic storage processes with Lévy input. J. Appl. Prob. 29, 396-403.

[8] Pakes, A. G. (2004). Convolution equivalence and infinite divisibility. J. Appl. Prob. 41, 407-424. 